\title{
Conceptual Marxism and Truth
}

\author{
Inquiry Symposium on Kevin Scharp's Replacing Truth \\ Patrick Greenough, University of St Andrew \\ Version sent to Inquiry, 27th October 2015
}

\section{Preamble.}

Should philosophy be content to describe the actual structure of our thought and talk, or should it be concerned with producing a better structure? ${ }^{1}$ Put another way, should philosophy be in the business of giving a theory of our ordinary, everyday concepts or should it also be in the business of producing new and better concepts with which to build a theory of the world and our place in it? At the extreme right-wing, is Conceptual Conservatism, the view that our everyday concepts are perfectly in order as they are: philosophy should leave everything just as it is. ${ }^{2}$ In the centre-left, is Conceptual Explication, the view that our everyday concepts are typically too rough-hewn as they stand to feature in rigorous philosophical theory. Such concepts need dressing and polishing if they are to be fit for the building of theory. ${ }^{3}$ At the extreme left, is Conceptual Marxism, the view that many (if not most) of our ordinary concepts are, for various reasons, unfit for bearing the load of useful philosophical explanations. Such concepts should be removed-at least in the regions where they prove to be troublesome or defective. The purpose of philosophy, on such a view, is not to merely interpret our concepts but to replace them. ${ }^{4}$

Kevin Scharp is a Conceptual Marxist of a rather extreme kind. In Replacing Truth (2013), the concept of truth is taken to be fundamentally incoherent. ${ }^{5}$ As such, Scharp reckons it to be unsuited for systematic philosophical theorising and in need of replacement-at least for regions of thought

\footnotetext{
${ }^{1}$ Cf. Strawson (1959, p. 9).

${ }^{2}$ Even if it turns out that our ordinary concepts are the source of all philosophical paradox. The most extreme exemplar of this view is Wittgenstein (1953) I.124; see also I.133.
}

${ }^{3}$ See Carnap (1950). Haslanger (2006, pp. 95-6) takes her "ameliorative project" to be offering Carnapian explications of gender and race concepts.

${ }^{4}$ Conceptual Marxists include Tarski $(1936,1944)$ and Scharp (2013) on truth, and Schiffer (1996) and Weiner (2009) on knowledge.

${ }^{5}$ All page references are to this book. See also Scharp (2007, 2012). Scharp speaks of "conceptual inconsistency" rather than "conceptual incoherence". I use the latter expression here and below as it can cover a broader range of views including the view whereby the constitutive principles of incoherent concepts do not entail any false proposition, but merely some proposition which is not assertible. 
and talk which permit liar sentences and their ilk to be formulated. ${ }^{6}$ This replacement methodology is radical because it not only recommends that the concept of truth be replaced (in troublesome domains) but that the word "true" be replaced too. Only Tarski has attempted anything like it before. ${ }^{7}$ In assessing Replacing Truth below, a lot of what I have to say concerns the credentials of the replacement methodology on offer, though I also connect various features of this methodology to the details of Scharp's own solution to the liar paradox. My goals are four-fold: to summarise the many components of Scharp's theory of truth; to highlight what I take to be some of the excess baggage carried by the view; to assess whether, and to what extent, the extreme methodology on offer is at all called for; finally, to briefly propose a less radical replacement strategy for resolving the liar paradox. To keep things focussed, I have organised the main discussion around a set of problems / questions. ${ }^{8}$

\section{Scharp's Theory of Truth: The Thumbnail Version.}

Scharp's theory of truth has many interlocking parts, which can be summarised as follows:

(a) Truth is an incoherent concept; the liar paradox demonstrates this (Ch. 3-4, esp. 57-62). ${ }^{9}$

(b) A concept is incoherent if and only if its constitutive principles (jointly) have false consequences. As such, one or more or these constitutive principles must be false (pp. 36-43).

Cartoon Exemplar One: (i) "is an oldster" applies to $\mathrm{x}$ if $\mathrm{x}>60$, (ii) "is not an oldster" applies to $\mathrm{x}$ if $\mathrm{x}$ $<65$. So, consider Charlie who is 62. The constitutive principles for "oldster" entail that Charlie is an oldster and that Charlie is not an oldster. So, they entail something necessarily false-a contradiction.

${ }^{6}$ Eklund (2002) also takes the concept of truth to be incoherent but does not recommend replacement. Equally, Dummett (1975) thinks that vagueness infects language with a kind of incoherence but holds that such incoherence is irremediable-we just have to live with it.

${ }^{7}$ See, e.g., Tarski $(1944, \S 14)$ on his hypothetical world congress on "frue" and "true". Confession: I found it a pity that the connections and differences between Tarski and Scharp did not get discussed in Replacing Truth.

${ }^{8}$ Replacing Truth is novel, ambitious, broad in scope, packed with arguments and insights, technically adept, timely, important, and clearly written. I cannot hope to evaluate the myriad features of the view here. Nonetheless, I hope to have put my finger on its many virtues and, what I take to be, its main vices.

9 That truth is an incoherent concept was first proposed by Tarski $(1933,1944)$. More recent proponents include Yablo (1993a, 1993b), Ludwig (2001), Badici and Ludwig (2007), Eklund (2002a, 2002b, 2005, 2007), Patterson (2006, 2007, 2008, 2009); cf. Priest (1979, 2006). See Scharp (2013, ch. 5) for relevant discussion. 
Cartoon Exemplar Two: (i) "is a blair" applies to $\mathrm{x}$ if $\mathrm{x}$ is a chair, (ii) "is not a blair" applies to $\mathrm{x}$ if $\mathrm{x}$ is blue. The constitutive principles for "blair" entail something contingently false: that there are no blue chairs (cf. Scharp's term "rable", p. 36). ${ }^{10}$

(c) A principle $p$ is constitutive for a concept $C$ if and only if competence with $C$ entitles one to accept $p$ (or would entitle one to accept $p$ when there is no countervailing evidence), (pp. 46-7). ${ }^{11}$

(d) The central constitutive principle for truth is the T-schema: a sentence $S$ (which says that $p$ ) is true if and only if $p$, (p. 16, pp. 62-3). (The corresponding rules of truth-introduction and truthelimination are just as central.)

(e) This schema is an incoherent constitutive principle: it entails a contradiction when applied to the liar sentence $\mathrm{L}$, where $\mathrm{L}=$ " $\mathrm{L}$ is not true" (given a suitably powerful logic such as intuitionistic or classical logic) (pp. 99-100).

(f) The standard liar paradox is blocked because the T-schema is taken to be (in some sense) invalid (p. 101).

(g) Without the T-schema, the predicate "is true" cannot function as a (general) device for endorsement and rejection (pp. 62-64). ${ }^{12}$

(h) So, Scharp concludes, the concepts of truth is severely defective and must be replaced (Ch. 5, esp. pp. 137-145).

(i) Moreover, the truth-predicate must be replaced too: we must learn not to use "true" anymore (at least for the scientifically respectable fragments of the language in which the liar paradox can be formulated), (pp. 142-143, pp. 275-277).

10 Scharp (pp. 37-8) additionally offers a non-cartoon candidate incoherent concept, namely mass (as employed in Newtonian mechanics). Such a concept was replaced by two concepts: proper mass and relativistic mass (which, despite their names, are not to be seen as kinds of mass).

${ }^{11}$ Here the notion of entitlement is an externalist, species of default warrant of the kind sponsored by Burge (1992). The qualifier "would entitle one to accept $p$ when there is no countervailing evidence" is included in order to address the worry that once exposed to the liar paradox we obtain a conclusive defeater for being entitled to accept the T-schema (assuming the credentials of the logic employed are not in question). One worry with such a weakened view of constitutive principles is that a competent subject need have no disposition to accept a constitutive principle for truth-but then we lose a general account as to why the liar paradox is so seductive. Eklund's conception of constitutive principles, in contrast, entails that a speaker is disposed to accept the constitutive principle as true which provides him with an explanation as to why the paradox exerts what he terms "pull" (see Eklund 2002a).

${ }^{12}$ Here the thought is that the truth predicate has two broad functions. Suppose I decide to endorse something that you said when we met yesterday at Noon but I cannot remember exactly what it was that you said. The truth-predicate allows me to say (or just merely believe) the following: what you said yesterday at Noon is true. This counts as an indirect endorsement of what you said. Likewise, if I want to indirectly reject what you said I can say or believe: what you said at Noon was not true. 
(j) Crucially, truth is not to be replaced with a single new concept. That is, a Mono-Replacement Strategy is not called for because the replacement concept would be too weak to be both consistent and equipped to take on the functional role of truth (p. 148). Rather, truth is to be replaced with two concepts which each divide up the main functional roles of truth between them: a Bi-Replacement Strategy (p. 149).

(k) The replacement concepts are: descending truth and ascending truth. The replacement theory is thus the combined theory of ascending and descending truth (ADT), (pp. 149-154).

(1) The (central) constitutive principle for descending truth is the following disquotational principle: If a sentence $S$ (which says that $p$ ) is descending true then $p$, (p. 149). (The corresponding elimination rule is also valid.)

(m) The (central) constitutive principle for ascending truth is the following enquotational principle: If $p$ (and a sentence $S$ says that $p$ ) then $S$ is ascending true, (p. 149). (The corresponding introduction rule is also valid. $)^{13}$

(n) The predicate "is descending true" behaves (very) roughly like "is necessarily true", while "is ascending true" behaves (very) roughly like "is possibly true".

(o) However, descending truth is not closed under validity or replacement of logical equivalents. (Background: validity is not necessary truth-preservation for Scharp (pp. 149-152, pp. 172-3) following the lead of Field (2006).)

(p) A sentence $S$ is safe if and only if: if $S$ is ascending true then $S$ is descending true. So, $S$ is unsafe if and only if $S$ is ascending but not descending true, (pp. 152-153).

(q) Liar-like sentences, and their negations, are unsafe (pp. 173-177). So, liar sentences, and their negations, are ascending true, but not descending true.

(r) The descending liar paradox (which invokes the descending liar identity DL = "DL is not descending true") is blocked because descending truth does not validate the following enquotational principle: if $p$ (and a sentence $S$ says that $p$ ) then $S$ is descending true, (pp. 173-4). (The corresponding introduction rule is also not valid.)

(s) The ascending liar paradox (which invokes the ascending liar identity AL = "AL is not ascending true") is blocked because ascending truth does not validate the following disquotational principle: If $S$ is ascending true (and $S$ says that $p$ ) then $p$, (pp. 173-4). (The corresponding elimination rule is also not valid.)

(t) The upshot is that classical logic is preserved in the replacement language; the replacement language is semantically closed; there are no strengthened liars (pp. 169-177).

${ }^{13}$ ADT has other constitutive principles (e.g. " $\mathrm{S}$ " is not ascending/ descending true if and only if "not-S" is ascending/descending true) but these are less central or derivative or both (p. 154). 
(u) Descending truth takes over truth's role as a device for (indirect) endorsement. Ascending truth takes over truth's role as a device for (indirect) rejection (p. 174). This is what makes the proposal a Bi-Replacement Strategy. ${ }^{14}$

(v) The replacement concepts also feature in the constitutive principles for: predication, reference, validity, meaning, assertion, provability, and kindred concepts (Ch. 8).

(w) For Scharp, a theory of truth not only has a prescriptive part but a descriptive part. The former is the replacement theory of ascending and descending truth; the latter is an account of what we were doing all along when using "true".

(x) For the descriptive theory, Scharp invokes Truth-Relativism and treats "is true" as assessmentsensitive. This descriptive theory employs the replacement notions and thus the prescriptive theory is prior to the descriptive theory (Ch. 9). ${ }^{15}$

\section{Three Initial Questions.}

One of the most interesting features of Replacing Truth is the metaphilosophy which underpins the whole project. A tantalising glimpse of this metaphilosophy is revealed in the following remarks:

My view is that philosophy is, for the most part, the study of inconsistent concepts [...] Once enough progress has been made to arrive at a set of relatively consistent concepts for some subject matter, it gets outsourced as a science (Scharp 2013, p. 3).

But these remarks raise three immediate problems:

Problem One: The replacement methodology on offer could be seen as a description of past (good) philosophical practice or it could itself be seen as a prescription for solving philosophical problems by replacing them. The first interpretation seems to be false. A great deal of extant philosophy has

14 There is a serious worry whether the descending/ascending truth-predicates can function as devices for generalised endorsement/rejection respectively. For example, consider the claim: every claim in Scharp's book is descending true. Here we are using the descending truth predicate to record our assent to all the claims made in Replacing Truth. One of these claims is: the descending liar sentence is not descending true. (Indeed, this sentence is a theorem of ADT.) Since I endorse this sentence, and descending truth is the device for endorsement, then I am committed to: "the descending liar sentence is not descending true" is descending true. But this claim quickly gives rise to paradox. (Cf. Scharp's discussion on pp. 286-7.) This is arguably just an instance of a more general worry that descending truth and ascending truth cannot, after all, function as consistent devices for endorsement and rejection, respectively. Scharp (pp. 280-1) acknowledges this worry but replies that "[...] there is no such thing as a consistent device for endorsement. Descending truth is as close as one can get without having an inconsistent concept" (p. 281). In effect, the concept of endorsement needs replacing with a new concept endorsement* Likewise, rejection needs replacing with rejection*. (It would have been useful to have seen this aspect of the theory spelt out in a bit more detail.)

15 This seems to be a key virtue of Scharp's view because if truth is defective then how can it be used to give a semantic theory for itself? (pp. 134-5). 
been conducted in a descriptive vein. The latter interpretation reveals some of the Marxist credentials of the project: philosophy needs to change in order to make progress. Which interpretation does Scharp have in mind? (I presume the latter.)

Problem Two: Concepts can be defective in manifold ways. They can be intensionally defective: incomplete, confused, unsatisfiable, or even incoherent/inconsistent. They can be extensionally defective: too inclusive, too narrow, empty, or divided of reference. They can be too complex, too simple, too unspecific, or too vague. They can be too parochial or too elitist. They can be redundant or not fit to feature in any useful explanation. They can be superseded, hackneyed, or systematically misapplied. They can be loaded with ideological baggage or serve as ongoing devices for deceit, discrimination, or oppression. Given all this, conceptual incoherence is just one source of conceptual malfunction, and perhaps not the most prevalent or interesting source. So, why take philosophy to be mainly concerned with concepts which are defective in only one way-by being incoherent? ${ }^{16}$

Problem Three: Even if a replacement methodology for philosophy is called for, then philosophy will be concerned to offer replacement concepts across a wide variety of domains, both scientific and non-scientific. Take the concept of responsibility. If philosophy is in the business of replacing this concept with a better one, then we would surely pass the replacement on to legal theory (and related domains of study), and not to science. Likewise if the concepts of right, law, artwork, family, convention, freedom, for example, are to be replaced then we would not outsource the replacements to the branches of science. In other words, Scharp's replacement methodology, as stated, has some significant baggage: scientism. Does Scharp really conceive of philosophy in the Quinean tradition as the mere handmaiden to science or will he allow a more inclusive view? ${ }^{17}$

\section{Deflationism and Replacement.}

Who can avail themselves of Conceptual Marxism? Not everybody, thinks Scharp. In particular, it is not available to the deflationist. He says (2013, p. 281):

The replacement strategy depends on truth's explanatory role-otherwise there is no reason to replace truth. So, deflationists have no reason to accept this view.

\footnotetext{
16 Scharp invokes Blackburn's phrase "conceptual engineering" to describe either a revisionary (light-duty) or replacement ("heavy-duty", p. 11) methodology for defective concepts (see Blackburn 2001, pp. 1-2; Scharp, p. 11). But even given this, Scharp has a far too restricted view of what Conceptual Engineering should be. For a general assessment of Conceptual Engineering, see my "Conceptual Engineering Without Concepts (and Without Engineering)."
}

${ }^{17}$ Cf. Strawson (2011) on two conceptions of philosophy. Quine (1960) wanted to replace the traditional notion of synonymy (and with it the notions of meaning and analyticity) with what he took to be the scientifically respectable notion of stimulus-synonymy (though Quine did not believe in concepts in anything like there traditional sense). Replacing Truth seems to be written in a very Quinean spirit (see esp. Ch. 7). 
Problem Four: Suppose one thinks that truth has an expressive but no explanatory role: truth is simply a device of disquotation, enquotation, and generalisation as encoded in the Tarskian Tschema for truth (and cognate schemas). Such is deflationism about truth. However, suppose the deflationist thinks that this device over-reaches: it doesn't do well when it comes to a range of paradoxical sentences such as the liar sentence. In the face of such paradox, one live option is to replace this device with a pair of devices, namely ascending and descending truth, together with the predicates "is ascending true", "is descending true". These replacement predicates, following Scharp, are fit to perform the expressive roles of the original truth-predicate. However, given deflationism, they are not fit for substantial philosophy theory-but that ought not to be a necessary condition for a replacement methodology. So, this seems to me to represent a perfectly coherent deflationary form of Conceptual Marxism. So, why exactly does Scharp think that a replacement strategy is inimical to deflationism?

\section{Individuating Concepts.}

What are concepts and how should we individuate them? The official line in Replacing Truth is that a replacement strategy is largely neutral on the metaphysics of concepts. ${ }^{18}$ However, it's hard to see how such neutrality can be upheld. Indeed, it would seem that the replacement strategy on offer is knee-deep in metaphysical commitments. In particular, it is clear, though not I think explicitly stated, that Scharp's Conceptual Marxism is committed to something like the following individuation principle:

Concept Identity Principle: Concepts are individuated by their constitutive principles. ${ }^{19}$

So, if two concepts differ in respect of their (central) constitutive principles, then they are different concepts. ${ }^{20}$ For example, if one concept has a truth-introduction rule as one of its central constitutive principles and another concept lacks such a rule then these cannot be the same concept. This is just why, for example, truth and ascending truth are different concepts. This has to be so for Scharp's replacement strategy to get off the ground in the first place.

Problem Five: Whether or not one accepts the Concept Identity Principle will be greatly influenced by the stand one takes on what kind of thing concepts are, where they live, and how they survive. Suppose concepts are three-dimensional enduring entities. Suppose further that a concept can

${ }_{18}$ Concepts might be abilities or capacities, mental representations, or abstract objects (see Scharp p. 35). Scharp is happy to be non-neutral about the epistemology of concepts-specifically their possession conditions.

${ }^{19}$ Scharp is, I think, also committed to mereological essentialism for concepts: if a concept $\mathrm{x}$ has some constitutive principle $\mathrm{y}$ as a part then $\mathrm{x}$ essentially has $\mathrm{y}$ as a part.

${ }^{20}$ I assume a difference in a peripheral constitutive principle need not make for a difference in concept. 
persist through a significant revision to one or more of its core constitutive principles. ${ }^{21}$ Then the Concept Identity Principle is false. In effect, Scharp (implicitly) assumes that an endurantist view of concepts is wrong. Equally, one might take concepts to be four-dimensional perduring entities with temporal parts, where these temporal parts are composed of different sets of constitutive principles. On such a view, the Concept Identity Principle needs re-working such that a concept is individuated by its temporal parts, and temporal parts are individuated via the set of constitutive principles true of the concept in question at a particular time. Scharp (implicitly) rejects such a perdurantist view since it permits conceptual persistence through a perdurantist conception of change-namely, whereby the concept is an aggregate of temporal parts, where these parts may be composed of different sets of (core) constitutive principles. ${ }^{22}$

The problem for Scharp, then, is not so much that he is far from neutral on the metaphysics of concepts, but rather that he does not address the arguments for or against the various ways in which concepts are to be individuated. This is no mere quibble. It lies as the heart as to whether incoherent concepts call for mere conceptual revision (light-duty Conceptual Engineering) or for the much more radical Marxist methodology of conceptual replacement (heavy-duty Conceptual Engineering). Conceptual revision of truth, in one prominent form at least, would involve mere replacement of the constitutive principles for the concept of truth, but the concept itself could survive such revisions, and we can thus continue using the same concept-word to pick out the revised concept-even if the revision is of a core principle. ${ }^{23}$ The replacement strategy, meanwhile, entails that such revision means that we are dealing with a new concept—that much is guaranteed by the Concept Identity Principle, to be picked out by a new concept word. But why think that? Just as words can survive substantial changes in their meaning, then why cannot concepts survive substantial changes in their (core) constitutive principles?

\section{Provability.}

Just how radical is Scharp's replacement strategy? Very. Far more radical than is acknowledged in Replacing Truth. This is revealed when we consider the relationship between the concept of truth and other concepts such as provability, assertion, belief, knowledge, and more. Take the concept of

${ }^{21}$ To make sense of such a view, one might well time-index the set of constitutive principles. On such a view, the Concept Identity Principle is now valid when stated as follows: concepts are individuated via their time-indexed sets of constitutive principles. This form of endurantism is also (implicitly) rejected by Scharp because it permits conceptual persistence through a kind of changebeing composed of the time $\mathrm{t} 1$ set of time constitutive principles, and being composed of the time $\mathrm{t} 2$ set of constitutive principles.

${ }^{22}$ Cf. Kaplan (1990) on the metaphysics of words.

${ }^{23}$ If conceptual revision is all that is called for then the title of Kevin's book would be the rather more humdrum: Replacing the Constitutive Principles for Truth (but Keeping the Concept of Truth and the Predicate "is true"). See below. 
(informal) proof and (informal) provability. With respect to these notions, Scharp recommends that we replace the following constitutive principle connecting provability and truth: ${ }^{24}$

(P1) If " $\mathrm{S}$ " is provable then " $\mathrm{S}$ " is true

with:

(P2) If "S" is provable with "S" is ascending true. ${ }^{25}$

Problem Six: Principle P1 is a constitutive principle for provability. To replace this principle with P2, while keeping the concept of provability unchanged, is to be engaged with conceptual revision and not conceptual replacement, as we have just seen. The Concept Identity Principle enforces the result that to replace P1 with P2 means that we are now dealing with a different concept of provability. Crucially, we are not allowed to use the word "provable" to pick out this new concept, so we have to introduce some new vocabulary to refer to it. So, the replacement principle should in fact be:

(P3) If " $\mathrm{S}$ " is provable ${ }_{1}$ then " $\mathrm{S}$ " is ascending true,

where "provable 1 " picks out the replacement concept of provability. ${ }^{26}$ This begins to reveal how extreme Conceptual Marxism really is: replace the concept of truth and you must also replace the concept of provability, and indeed you must also replace the word "provable" too. Does Scharp acknowledge that this is an unavoidable feature of his view, a feature which shows that the view is more radical than the view officially advertised in Replacing Truth?

\section{Belief, Assertion, Correct Belief, and Correct Assertion.}

The revolutionary nature of Conceptual Marxism does not stop there. Scharp offers the following replacement principles for belief, assertion, correct belief, and correct assertion (p. 207, pp. 215-16):

(AB1) An assertion/belief that $p$ is ascending true if things are as they are asserted/believed to be (with respect to this assertion/belief).

(AB2) An assertion/belief that $p$ is descending true only if things are as they as they are assertion/ believed to be (with respect to this assertion/ belief).

(AB3) It is correct to assert/believe that $p$ only if $p$ is ascending true.

(AB4) It is correct to assert/believe that $p$ if $p$ is descending true.

24 See p. 205.

${ }^{25}$ Scharp cannot use Descending Truth as here as that would quickly leads to contradiction given that the Descending Liar Sentence is provable on no assumptions.

${ }^{26}$ Indeed, the new concept may not be a concept of provability at all, just as ascending and descending truth are not concepts of truth but surrogates for truth. 
Problem Seven: However, by Scharp's lights, these cannot be the right replacement principles for (correct) assertion/ belief. That is because, again, the Concept Identity Principle enforces the result that to replace a constitutive principle for a concept is to replace the concept. So, we can no longer use the same concept-word to pick out the new replacement concept. So, Scharp's replacement principles should be:

(AB5) An assertion ${ }_{1} /$ belief $_{1}$ that $p$ is ascending true if things are as they are asserted ${ }_{1} /$ believed $_{1}$ to be.

(AB6) An assertion ${ }_{1} /$ belief $_{1}$ that $p$ is descending true only if things are as they are asserted ${ }_{1} /$ believed $_{1}$ to be .

(AB7) It is correct to assert ${ }_{1} /$ believe $_{1}$ that $p$ only if $p$ is ascending true.

(AB8) It is correct to assert ${ }_{1} /$ believe $_{1}$ that $p$ if $p$ is descending true,

where "assertion," and "belief ${ }_{1}$ " pick out the replacement concepts for assertion and belief, respectively. Surely Scharp is committed to the more radical revision resulting in AB5-8, rather than AB1-4?

Problem Eight: The general lesson ought to be clear. If we replace the concept of truth with one or more surrogate concepts then any concept which is constitutively linked to truth via one of its core constitutive principles must be replaced too. Furthermore, we cannot use the old concept-word to pick out this new concept-we must introduce a new concept-word to refer to it. The problem is that not only is the concept of truth constitutively linked to the concepts of provability, assertion, and belief, it is also so linked to myriad other concepts such as the concepts of inquiry, objectivity, reality, knowledge, judgment, evidence, justification, confirmation, probability, fact, being, truthvalue, truth-bearer, reference, denotation, satisfaction, truth-condition, meaning, content, proposition, representation, necessity, possibility, contingency, and more. In turn these concepts are constitutively linked to a wider class of concepts which may well include, in the end, all concepts of central philosophical interest. All these concepts must be replaced too, together with their respective concept-words. The problem faced by Scharp thus proliferates very quickly. Again, this not only makes the view much more radical than the advertised view in Replacing Truth, it begins to make the view implausible. Would it not be better to ditch the Concept Identity Principle altogether and go for a less extreme replacement strategy whereby we can keep the old concept and the old concept-word, but merely replace the constitutive principles? Surely we should explore a more moderate left-wing option first before trying out the wholesale conceptual cleansing recommended by Scharp? (See below.)

\section{The Scope Problem: the Case of Vagueness.}

Scharp's replacement strategy for incoherent concepts only becomes a genuine contender when we are given an account of how it would play out with respect to concepts other than truth but which, 
by Scharp's lights, are incoherent concepts that prove to be the source of philosophical paradox. What would a replacement strategy for vagueness look like? Here we can adapt the account of vagueness given by Eklund (2002a). The rough idea would be that one of the core constitutive principle for the vague concept yellow would be a principle of tolerance as follows:

(TOL): For all $x$, if $x$ is yellow then $x^{\prime}$ is yellow,

(where $x^{\prime}$ is the successor of $x$ in a sorites series). TOL plus the fact that the first patch in the series is yellow, and the last is not, entail a contradiction, at least given some suitably strong logic. Since TOL is highly plausible, and the logic initially seems to be in good order, we have a paradox. Eklund's response to this paradox is to hold that TOL is a constitutive principle for the concept yellow and that TOL is false- that just why the concept yellow is an incoherent concept since it is governed by constitutive principles which jointly entail something false. ${ }^{27}$

Problem Nine: The replacement version of Eklund's view would resolve the sorites paradox via conceptual replacement. However, to effect such a replacement, the principle TOL would have to be replaced with some principle which was suitably weakened so as not to give rise to the paradox. ${ }^{28}$ Moreover, such a replacement principle would ensure that we are no longer dealing with the concept yellow, but a new concept-to be picked out by a new concept-word such as "yellow ${ }_{1}$. So much is enforced by the Concept Identity Principle. But then in order to address the sorites paradox in English, we need to replace every single vague concept with a new concept, and replace every single vague predicate in English with a new predicate which picks out this new concept. Unlike the liar paradox, which only arises when we formulate a liar sentence, vagueness is ubiquitous in natural language. So the required replacements are not simply limited to some special region of thought and talk. Upshot: once Conceptual Marxism is applied to vagueness the result is an even more extreme kind of conceptual cleansing. Scharp is then faced with a dilemma: either treat vagueness differently from how he treats the liar paradox and kindred paradoxes (and face a charge of ad hocness) or treat vagueness via his Conceptual Marxism (and face a charge of wholesale conceptual cleansing). Which horn of the dilemma will he take?

\section{The Knower Paradox.}

Is the concept of knowledge incoherent? Scharp's (final) analysis of knowledge is: $S$ knows that $p$ if and only if $S$ believes $p, S$ is justified in believing that $p$, and $p$. Since this analysis does not employ truth then it does not need replacement as it stands. ${ }^{29}$ However, one version of the knower paradox

27 One attraction of such a view is that it preserves classical logic.

${ }^{28}$ One extra worry here is: what would the replacement principle of tolerance look like and could ordinary users of English deploy it successfully? (See the worries raised by Dummett 1975.) One candidate principle might be a contextualised form of the tolerance principle whereby adjacent patches are never category different-at least when viewed together (see Raffman 1994; cf. GraffFara 2000). I eagerly await Scharp's book Replacing Tolerance to see how the view pans out!

${ }^{29}$ Setting aside issues which arise from Gettier cases. 
of Kaplan and Montague (1960) surely shows that knowledge is an incoherent concept, if truth is. ${ }^{30}$ A Scharp-style replacement strategy for the knower paradox would involve replacement of one, or more, of the constitutive principles for knowledge. The two main culprits in the proof are a factivity principle and a necessitation rule (assuming the logic is in good order):

(K-Fact): $\quad \vdash$ If " $\mathrm{S}$ " is known then $S$.

$(\mathrm{K}-\mathrm{Nec}) \quad$ From $\vdash \mathrm{S}$ infer $\vdash$ "S" is known. ${ }^{31}$

What kind of replacement solutions are available to Scharp? Consider the following two options:

Option One: (i) Retain K-Nec. (ii) Invoke Scharp's replacement theory of truth, specifically ascending truth, and replace K-Fact with:

(K1-Fact) $\vdash$ If $S$ is known then $S$ is ascending true.

Without K-Fact, the knower is blocked. (And we cannot derive K-Fact from K1-Fact, as ascending truth does not validate a descending truth-elimination rule.)

Option Two: (i) Retain K-Fact. (ii) Invoke Scharp's replacement theory of truth, specifically descending truth, and replace K-Nec with:

$(\mathrm{K} 1-\mathrm{Nec})$ From $\vdash$ " $\mathrm{S}$ " is descending true infer $\vdash$ " $\mathrm{S}$ " is known.

Without K-Nec, the knower is blocked. (And we cannot derive K-Nec from K1-Nec, as descending truth does not validate necessitation for descending truth.)

However, neither of these options is available to Scharp given his commitment to the Concept Identity Principle: as we have seen, to replace a core constitutive principle for knowledge, is to replace the concept of knowledge and so one needs a new concept-word to pick out this new concept. $^{32}$ Scharp can, however, avail himself of one of the following two options:

Option Three: A Mono-Replacement Strategy whereby the concept of knowledge is replaced with the concept of knowledge ${ }_{1}$ which has the constitutive principles:

(K2-Fact) If " $\mathrm{S}$ " is known 1 then $\mathrm{S}$ is ascending true.

30 Scharp, fn. 20, p. 216 says "it might well be".

31 Proof: Suppose "k is not known" is known. By K-Fact, k is not known. But from this same supposition, given $\mathrm{k}=$ " $\mathrm{k}$ is not known", via substitution, $\mathrm{k}$ is known. Contradiction. Conclude, via reductio: " $k$ is not known" is not known. Via a further application of substitution, we can then derive: $\mathrm{k}$ is not known. Since this is rigorously provable on no assumptions, then we can also derive, via $\mathrm{K}-\mathrm{Nec}$, that " $\mathrm{k}$ is not known" is known. Contradiction.

32 Scharp cannot adopt a hybrid strategy whereby some concepts get replaced and other concepts get revised—so much is enforced by the Concept Identity Principle. 
$(\mathrm{K} 2-\mathrm{Nec})$ From $\vdash \mathrm{S}$ infer $\vdash$ " $\mathrm{S}$ " is known $_{1}$.

Option Four: A Mono-Replacement Strategy whereby the concept of knowledge is replaced with the concept of knowledge ${ }_{2}$ which has the constitutive principles:

(K3-Fact) If "S" is known $_{2}$ then $S$.

$(\mathrm{K} 3-\mathrm{Nec})$ From $\vdash$ " $\mathrm{S}$ " is descending true $\mathrm{S}$ infer $\vdash$ " $\mathrm{S}$ " is known $_{2}$.

Here "known 1 " and "known 2 " pick out new surrogate concepts for knowledge.

Finally, as it turns out, Scharp could seek to address the knower paradox via a Bi-Replacement Strategy, as follows:

Option Five: Just as with truth (and reference and predication), there is a Bi-Replacement Strategy whereby we have: two replacement concepts, namely Ascending Knowledge and Descending Knowledge. The core constitutive principle for descending knowledge is:

(K4-Fact) If "S" is descending known then S.

The core constitutive principle for Ascending Knowledge is:

(K4-Nec) From $\vdash \mathrm{S}$ infer $\vdash$ "S" is ascending known.

This option differs from the previous two options because it does not invoke the concepts of ascending or descending truth. ${ }^{33}$

Problem Ten: Which of these three options should Scharp choose? Given that he already has the concepts of ascending truth and descending truth in hand to address the liar paradox, then it looks as if one of the Mono-Replacement strategies is the better option. The thought here is that a kind of maxim of minimal mutilation is in play: revise or replace as little as possible so as to save what can be saved of truth (and logic). Upshot: even though we need two new concepts to solve the liar, we don't need two new replacement concepts for knowledge to solve the knower (once we have two new replacement concepts for truth). Still, a residual problem remains: which of Option Three and Option Four are we to choose, and why?

I don't think is too much of a challenge since Conceptual Marxism should probably allow that sometimes replacement options can be equally good. Nonetheless, this worry opens up the question as to whether, once the maxim of minimal mutilation is on the table, we should be looking for a viable replacement strategy for truth which is less disruptive than a Scharp-style Bi-Replacement strategy. Let's see.

${ }^{33}$ It's worth noting that, on a Scharpian view, just as ascending truth and descending truth are not kinds of truth or new ways of thinking about truth (despite their names), all these replacement concepts for knowledge are not kinds of knowledge or even new ways of thinking about knowledge (despite their names). 


\section{Overkill.}

Both Eklund (2002a) and Scharp (2013) take the liar paradox to show the T-schema to be (in some sense) invalid, where the T-schema may be given as: $\mathrm{S}$ is true if and only if $p$ (where $\mathrm{S}$ says that $p$ ). However, we only need to reject (or indeed restrict) one direction of the T-schema in order to block the liar paradox. Eklund (2002a) does not tell us which direction we should reject (or restrict). Perhaps he thinks we should reject both directions. ${ }^{34}$ That is arguably a kind of overkill because the maxim of minimal mutilation counsels us to save what can be saved of the old theory of truth. So, if we can save one direction in the face of the liar paradox, then we should do so. Scharp meanwhile adopts a similar stance since neither direction of the T-schema for truth is preserved in his theory. Rather, as we have seen, the two directions of the T-schema are replaced with the the introductionrule for ascending truth, and the elimination rule for descending truth. Likewise, this is also arguably a kind of overkill because we only need to reject one direction of the T-schema to block the paradox-and so we only need to find one surrogate replacement principle to have a stable theory. ${ }^{35}$ We can do this in either of the following ways:

Option One: Reject the truth-elimination rule for truth and with it the following direction of the Tschema: If $S$ is true then $p$ (where $S$ says that $p$ ). Introduce a new concept-descending truth-which has an elimination rule (but not an introduction rule) which can play the role that truth's elimination rule played as a device for (indirect) assent, as when one says, e.g., "what she said was true".

Option Two: Reject the truth-introduction rule for truth and with it the following direction of the Tschema: if $p$ then $S$ is true (where $S$ says that $p$ ). Introduce a new concept-ascending truth-which has an introduction rule (but not an elimination rule) which can replace the role that truth's introduction rule played as a device for (indirect) denial, as when one says, e.g., "what he said what not true".

Problem Eleven: Which of these options should Scharp choose between? As it turns out, neither. Why is that? You've guessed it-it's because of our old friend The Concept Identity Principle. Once the elimination-rule for truth has been replaced, we are no longer dealing with the concept of truth. Likewise, for the introduction rule. In other words, there is no Mono-Replacement Strategy available not only for the concept of truth, but for the constitutive principles for truth. That's why Scharp's Conceptual Marxism is inevitably extreme-at least in so far as The Concept Identity Principle is taken to be inevitable. So, on pain of repetition, we may ask again: Is this Principle so inevitable?

${ }^{34}$ I'll come back to this below.

${ }^{35}$ Of course the liar paradox can be derived in different ways—so the issue is a bit more complicated. See below. 


\section{The Asymmetry of Truth-Introduction and Truth-Elimination.}

We have just seen how there is a much weaker replacement strategy available which does not involve replacing the concept of truth, but rather involves weakening, but keeping, the concept of truth and also invoking a new additional concept to make up for that weakness. Suppose, unlike Scharp, one were attracted to such a view. The question still remains: which of the two options canvassed above should be preferred? One thought might be that since the truth-introduction and truth-elimination rules are on a par in terms of plausibility then if we reject one rule, we must, by symmetry, reject the other. That would result in neither option being available and a Scharp-style BiReplacement Strategy would in the end be called for. However, such a symmetry argument is not available to Scharp for two reasons. Firstly, any prescriptive, replacement methodology should allow that we are simply free to choose how to replace the constitutive principles for truth in the event of a tie between two equi-plausible replacement strategies. We saw as much when it came to the kind of Mono-Replacement Strategy for replacing the concept of knowledge above. Secondly, the truth-introduction rule and truth-elimination rule, as it turns out, are asymmetrical on completely independent grounds. So, anybody wishing to restrict, revise, or replace the concept of truth, in the face of the liar paradox, can only do so in only one way. ${ }^{36}$ Why is that?

In the literature on the liar paradox, there has been far too much focus on two types of formulation. One formulation focusses on the T-schema and its liar-instance: "L is not true" is true if and only if $\mathrm{L}$ is not true. The other formulation proceeds as follows: suppose the $\mathrm{L}$ is true. By substitution, "L is not true" is true. By truth-elimination, $\mathrm{L}$ is not true. Contradiction. Via reductio on the initial supposition, we may infer that $\mathrm{L}$ is not true. By truth-introduction, " $\mathrm{L}$ is not true" is true. Via substitution, conclude that $\mathrm{L}$ is true. Contradiction. The latter formulation is to be preferred because it is more explicit and does not depend on the T-schema but simply on the corresponding elimination and introduction rules. More generally, the most important versions of any paradox are those which use the weakest resources. That's because such versions present us with the clearest picture as to what are the candidate basic sources of the malady. ${ }^{37}$

A better, and indeed perhaps canonical, formulation of the liar paradox does not employ the rule of truth-introduction at all but simply an Alethic Rule of Necessitation as follows:

(T-Nec): From $\vdash \mathrm{S}$ infer $\vdash$ "S" is true.

The proof then goes as follows: Suppose that "L is not true" is true. Given truth-elimination we can infer that $\mathrm{L}$ is not true. Given that $\mathrm{L}=$ "L is not true", and substitution, we can also infer from our

36 This lesson applies to those such as Horwich (1990) who recommend we restrict the T-schema to its unproblematic instances in order to combat the liar paradox. What Horwich should be doing is merely restricting one direction of the T-schema (given a plausible maxim of minimal mutilation). Likewise the same applies to Eklund (2002a). More generally, I do not think the lesson advanced in the main text has been generally discussed or understood.

${ }^{37}$ Of course, we can't always decide in advance which of a variety candidate presentations of a paradox employs the weakest set of principles because it is often a controversial matter as to whether one set of principles is weaker than some other set. 
initial supposition that $\mathrm{L}$ is true. Contradiction. By reductio, infer that " $\mathrm{L}$ is not true" is not true. Another step of substitution gives us: $\mathrm{L}$ is not true. Using T-Nec, we can infer that " $\mathrm{L}$ is not true" is true. Contradiction on no assumptions. ${ }^{38}$

This represents a weaker, and so better, formulation of the liar paradox than the formulation which uses truth-introduction and truth-elimination because T-Nec is weaker than truthintroduction - and so merely restricting (or rejecting) the latter but not the former is insufficient to block the liar paradox. Upshot: insofar as truth-elimination and truth-introduction are relevantly symmetrical, then truth-elimination and T-Nec are relevantly asymmetrical because the latter is weaker than truth-introduction. So, if we were to reject or restrict either truth-elimination or T-Nec, it should be the truth-elimination rule. But then if that is so, then given the maxim of minimal mutilation, we should preserve not only T-Nec but the truth-introduction rule too. Our question has thus been answered: we should prefer Option One sketched above.

Final Problem: In other words, if one is tempted by some kind of replacement strategy, and constrained by a maxim of minimal mutilation, then one should seriously consider ditching the Concept Identity Principle so as to allow the following constellation of claims: keep the concept of truth in troublesome contexts; keep the word "true" in troublesome contexts; keep the constitutive principle of truth-introduction (and the alethic principle of necessitation T-Nec); but revise the concept of truth so it no longer validates a rule of truth-elimination; finally, use a replacement concept—namely, descending truth—-to function as a surrogate concept, equipped to function as a device for (indirect) assent. ${ }^{39}$ Surely this represent a less extreme, and more attractive proposal than the Bi-Replacement strategy offered in Replacing Truth? 4041

\footnotetext{
${ }^{38}$ For simplicity, I'm assuming that $\mathrm{L}=$ "L is not true" is a theorem.

${ }^{39}$ Cf. Friedman and Sheard (1987).
}

${ }^{40}$ My own view is that one viable solution to the liar paradox takes pretty much the shape just outlined, with one crucial difference: there is no need to view such a solution as representing any kind of replacement solution at all. How so? Well, the concept of truth does not need to be revised because it all along failed to validate a truth-elimination rule-it's just that we did not realise this. What is in need of revision is not the concept of truth, but our beliefs about what such a concept includes. Making good on that proposal, however, would take us too far afield—see Greenough ms "The Lazy Way with the Liar Paradox".

41 Versions of this material were presented at a session on Conceptual Engineering, Arché Graduate Conference, November 2014; Northern Institute for Philosophy, Aberdeen, December 2014; and at a Symposium on Replacing Truth, Eastern APA, Philadelphia, December 2014. Many thanks to the audiences and speakers at those events for very useful feedback-particularly JC Beall, Herman Cappelen, Toby Meadows, and Crispin Wright. Thanks also to my MLitt Class on the Philosophy of Logic, Autumn 2014, for much stimulating discussion of Replacing Truth. Particular thanks to Kevin Scharp for very valuable feedback on earlier versions. 


\section{References}

Badici, E. and Ludwig, K. (2007): "The Concept of Truth and the Semantics of the Truth Predicate", Inquiry, 50, pp. 622-38.

Beall, JC. (2008): Revenge of the Liar, Oxford: OUP.

Blackburn, S. (2001): Think, Oxford: OUP.

Burge, T. (1993): “Content Preservation”, Philosophical Review, 103, pp. 457-88.

Carnap, R. (1950): Logical Foundations of Probability, Chicago: University of Chicago Press.

Dummett, M. A. E. (1975): “Wang's Paradox", in Truth and Other Enigmas, 1978, Cambridge, MA: Harvard University Press.

Eklund, M. (2002a): “Inconsistent Languages”, Philosophy and Phenomenological Research, 64, pp. 251-75.

Eklund, M. (2002b): “Deep Inconsistency”, Australasian Journal of Philosophy, 80, pp. 321-31.

Eklund, M. (2005): “What Vagueness Consists In”, Philosophical Studies, 125, pp. 27-60.

Eklund, M. (2007): “Meaning-Constitutivity”, Inquiry, 50, pp. 559-74.

Field, H. (2006): “Truth and the Unprovability of Consistency”, Mind, pp. 567-605.

Friedman, H. and Sheard, (1987): "An Axiomatic Approach to Self-Referential Truth", Annals of Pure and Applied Logic, 33, pp. 1-21.

Graff-Fara, D. (2000): “Shifting-Sands: An Interest-Relative Theory of Vagueness", Philosophical Topics, 28, pp. $45-81$

Greenough, P., ms, “Conceptual Engineering Without Concepts (and Without Engineering)” Haslanger, S. (2006): "Philosophical Analysis and Social Kinds: What Good are Our Intuitions?", Proceedings of the Aristotelian Society, Suppl Vol., 80. p. 89-118.

Horwich, P. (1990): Truth, Oxford: Blackwells.

Kaplan, D. and Montague, R. (1960): “A Paradox Regained", Notre Dame Journal of Formal Logic, 1, pp. 79-90.

Kaplan, D. (1990): “Words”, Proceedings of the Aristotelian Society, Suppl Vol., 64, pp. 93-119.

Ludwig, K. (2007): "What is the Role of Truth in a Meaning Theory?", in Truth and Meaning: Topics in Contemporary Philosophy, New York: Seven Bridges Press.

Patterson (2006): “Tarski, the Liar, and Inconsistent Languages”, The Monist, 89, pp. 1-17. 
Patterson, D. (2007): “Inconsistency Theories: The Significance of Semantic Ascent”, Inquiry, 50, pp. $575-89$.

Patterson, D. (2008): “Understanding the Liar”, in JC Beall (ed.)

Patterson, D. (2009): "Inconsistency Theories of Semantic Paradox", Philosophy and Phenomenological Research, 79, pp. 387-422.

Quine, W. V. O. (1960): Word and Object, Cambridge, MA: MIT Press.

Raffman, D. (1994): “Vagueness Without Paradox”, The Philosophical Review, 103, pp. 41-74.

Scharp, K. (2007): “Replacing Truth”, Inquiry, 50, pp. 606-21.

Scharp, K (2012): “Truth, The Liar, and Relativism”, The Philosophical Review,

Scharp, K. (2013): Replacing Truth, Oxford: OUP.

Schiffer, S. (1996): “Contextualist Solutions to Scepticism", Proceedings of the Aristotelian Society, 96, pp. 317-33.

Strawson, P. F. (1959): Individuals: An Essay in Descriptive Metaphysics, London: Methuen.

Strawson, P. F. (2011): Philosophical Writings, Oxford: OUP, ch. 14.

Tarksi, A. (1933): “The Concept of Truth in Formalised Languages” in Logic, Semantics, MetaMathematics, translated by J.H. Woodier, edited by J.Corcoran, Indianapolis: Hackett.

Tarksi, A. (1944): “The Semantic Conception of Truth”, Philosophy and Phenomenological Research, 4, pp. 341-76.

Weiner, M. (2009): “The (Mostly) Harmless Inconsistency of Knowledge Attributions”, Philosophers' Imprint, 9, pp. 1-25.

Wittgenstein, L. (1953): Philosophical Investigations, Oxford: Basil Blackwells.

Yablo, S. (1993a): “Definitions, Consistent and Inconsistent”, Philosophical Studies, 72, pp. 147-75.

Yablo, S. (1993b): “Hop, Skip, and Jump: The Agnostic Conception of Truth", Philosophical Perspectives, 7, pp. 371-96. 